\title{
360-deg profilometry: new techniques for display and acquisition
}

\author{
Anand K. Asundi, MEMBER SPIE \\ C. S. Chan \\ M. R. Sajan \\ University of Hong Kong \\ Department of Mechanical Engineering \\ Hong Kong \\ E-mail: archana@hkusub.hku.hk
}

\begin{abstract}
Two optical methods are proposed for shape measurement and defect detection of curved surfaces in the form of a complete 360deg profile of the object. The first one is the standard structured light approach. Display of the resulting data is the emphasis of this section. The second approach uses modulated structured light with a scanning digital camera for faster and simpler data acquisition. Quantitative processing is done off-line while real-time moiré produces enhanced display of the defects for qualitative analysis.
\end{abstract}

Subject terms: profilometry; projected gratings; scanning moiré structured light; phase shifting; logical moiré.

Optical Engineering 33(8), 2760-2769 (August 1994).

\section{Introduction}

Structured light techniques are widely used for surface profilometry ${ }^{1-5}$ and defect detection. ${ }^{6,7}$ Light could be structured either as a single spot, a thin stripe, or multiple stripes. Each method has its own application niche and corresponding drawbacks and merits. The single-spot system requires scanning the beam in two perpendicular directions. In particular, if the object to be tested is moving such as in sheet metal inspection, scanning in one direction is sufficient. The scanned beam is recorded using a type of position sensing detector that enables measurement of difference in coordinates of spot from a reference. Adaptations of these systems can be found in ranging devices. Scanning systems of this type are generally complex but have been developed for specialized systems as a fast and accurate measurement tool. A simpler variant of the scanning system is the generation of a stripe of light. Although sophisticated line generators with focusing optics are available, a simpler system comprising a glass rod provides for adequate sensitivity in most applications. The versatility of the structured light system has made them a ubiquitous tool for profile measurement. However, they still require either the line of light to be scanned or, as is generally the case, the object to be mapped is on a stepped translation stage. To overcome the need for moving parts, projection of multiple stripes is used. ${ }^{8}$ When used in con-

Paper 36113 received Nov. 22, 1993; revised manuscript received Feb. 24, 1994; accepted for publication Feb. 25, 1994

(C) 1994 Society of Photo-Optical Instrumentation Engineers. 0091-3286/94/\$6.00. junction with phase stepping ${ }^{9}$ the method has sensitivities similar to the single-stripe method. Furthermore, because of recent developments using computer gratings and a liquid crystal display (LCD) projection system, a system can be developed with no moving parts. ${ }^{10}$

Most of the objects analyzed for surface profiling are what are commonly referred to as $2 \frac{1}{2} \mathrm{D}$ objects, i.e., the depth profiles are with respect to a plane or only one view of the imaging system is evaluated. For a complete $360-$ deg profile, similar principles as the single stripe can be and have been adopted. ${ }^{11-13}$ In this case, rather than translate the object, it is mounted on a rotational stage. Analysis of each stripe proceeds in much the same way as before and an "unwrapped" depth profile of the object is obtained. This method has been termed the 360-deg profilometric method. As with other single-stripe methods, this requires a step motor controlled rotary stage and generally each line is analyzed before the next rotational step. Data acquisition is thus slowed, especially when a preliminary inspection is sufficient and processing can be delayed off-line. This suggests a need for a multiple line generation scheme. Indeed, the earlier 360-deg profilometry scheme ${ }^{11}$ did use a projected grating to illuminate the object. Recording was accomplished using a linear photodiode array, thus analyzing a "single line" on the object at a time. Since phase shifting of gratings was used for analysis this process was even more time-consuming than the striped light scheme. However, this illumination and recording scheme has been shown ${ }^{14}$ to be able to record a horizontal grating on the unwrapped 360-deg surface that could be analyzed off-line. 
This paper further evaluates both these approaches. Using the striped light approach, the emphasis is primarily on the acquisition and data display techniques. These become more important, particularly when the unwrapped profile must be put back into its original form for display. The second scheme, that of projected gratings, has more scope for improvement. A novel scheme for generating cross-line projected grating is described. In addition, digital recording of the pattern enables a scanning moiré to be generated capable of highlighting deviations in the pattern for qualitative on-line defect assessment. The proposed system has a lot of potential for low-cost and rapid evaluation particularly when phase shifting is incorporated in the analysis.

\section{Striped Light System}

The basic configuration for 360-deg profilometry is similar to the system for the linear scanning system as mentioned earlier. The translational stage is replaced by a turntable that is driven by a stepping motor. The stepping motor is controlled by a driver connected to the $\mathrm{PC}$ via the parallel port. The image of the stripe recorded by the CCD camera is digitized using a PC-based frame grabber and stored in a frame buffer. A diode laser is used as the light source. A cylindrical lens is used to form the line of light. Figure 1 is the schematic of the $360-\mathrm{deg}$ profilometric setup. The emphasis in this system is on low cost but with sufficient sensitivity for application in the manufacturing industry.

Before fixing the object for testing, the diode laser must be properly aligned such that the line of laser light coincides with the axis of rotation of the turntable. For each particular configuration, a calibration must be performed before testing. For comparative analysis, a stepped cylinder of known dimensions is used for calibration. This test object should be clamped to the turntable such that the cylinder is concentric with the turntable's rotational axis. However, for shape measurement and defect detection, a stepped calibration strip that can be adhered to the test object itself is sufficient. During scanning, the laser source is kept stationary. The CCD camera captures the image of the light strip, which is distorted proportional to the depth of the object. Thus, knowing the amount of shift in the image of the light strip and the calibration factor, the surface profile can be obtained. To obtain the complete peripheral profile of the object, the object is rotated through $360 \mathrm{deg}$. The output data file is stored as an array of either cylindrical $(\theta y r)$ or cartesian $(x z y)$ coordinates.

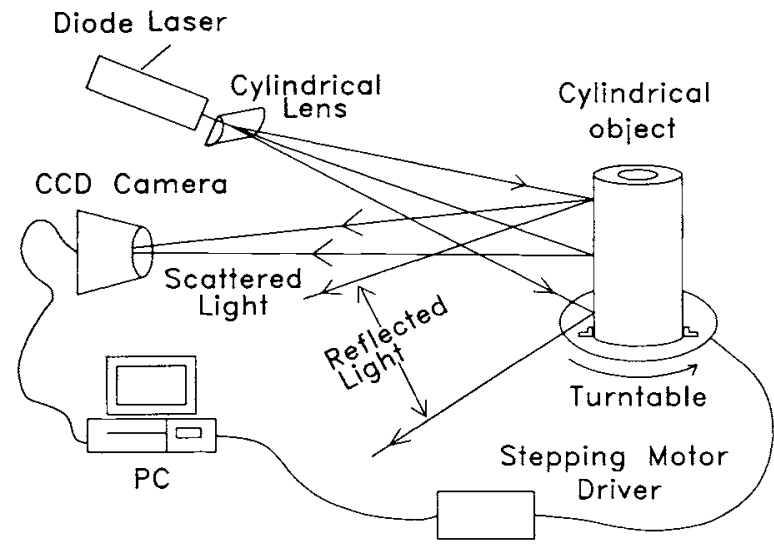

Fig. 1 Schematic of 360-deg profilometry using structured light.
Mesh size is determined by the product of the number of points per line and the number of rotational steps. The former is limited by the resolution of the camera while the latter is limited by the step angle of the stepping motor. These mesh sizes can be easily varied by altering either of these two parameters. In the current setup, the object is digitized to an image of $512 \times 512$ pixels and the smallest step angle of the stepping motor used to drive the turntable is $1.8 \mathrm{deg}$. However, a compromise has to be reached between the number of data points acquired and the speed of acquisition of the system. Another aspect contributing to the speed of the data acquisition phase is the scheme for determination of the position of the light stripe. Two commonly used approaches are the peak detection and the weighted centroid algorithms. The first detects the pixel or pixels with maximum intensity, following a global thresholding scheme. Sensitivity of 0.5 pixel is achieved, albeit under favorable conditions. The weighted centroid algorithm weights each pixel according to its intensity; 0.5-pixel sensitivity is assured with smaller sensitivities subject to cutoff values, stripe width, and noise. Figure 2 is a plot of the sensitivities achievable and is a result of 10 samples at each cutoff value. Larger cutoff values produce more repeatable results, but smaller values give higher sensitivity. A midway compromise giving 0.5-pixel sensitivity is adopted in this case.

A calibration strip of predetermined height steps was affixed to the test object and used for evaluating the system parameters. For the current results 50 angular positions were evaluated with each line sampled at 50 points resulting in 2500 data points. The time for data acquisition using the weighted centroid scheme on an IBM-PC 386 was about $24 \mathrm{~s}$ with a sensitivity of $0.10 \mathrm{~mm}$ in the depth direction. Data were stored as a matrix of points in columns of the angle $\theta$, the vertical height of the object $y$, and the depth profile $r$. An alternative scheme saves the data as $x(=r \cos \theta), y$, and $z(=r \sin \theta)$

\subsection{Experimental Results}

The first object used in this study was a cylindrical can dented at a location on the periphery. Unlike conventional tests, the surface of the can was not painted white but had the original markings, as shown later. This was all the more critical in this case since the background color of the can was brownish and the illuminated diode laser light was red. The recording was under dimmed room lighting but with no laser line filters attached to the camera. Figure 3 is the contour map and the topographic profile of the unwrapped periphery of the can. The dent is clearly identified, as is the calibration strip. Color coding of the contours is the next obvious display for ease of interpretation and is shown in Fig. 3(c). Finally, in the same vein one could use one of the other stereo vision display techniques such as the red/blue images or the random dot stereogram. (For a computer disk containing these images and other animated files please contact the authors.)

A 3-D wire diagram is another important display technique and the $\mathrm{PC}$ has the ability to manipulate these diagrams for a continuous visualization from a variety of angles. These could be recorded on video or animated within the computer! Figure 4 provides two views using this algorithm. Finally, slices of the can can be displayed at discrete heights if the data are saved in the $(x, y, z)$ format (Fig. 5). This aids in the detection of defects; a "perfect" cylinder gives a circular 


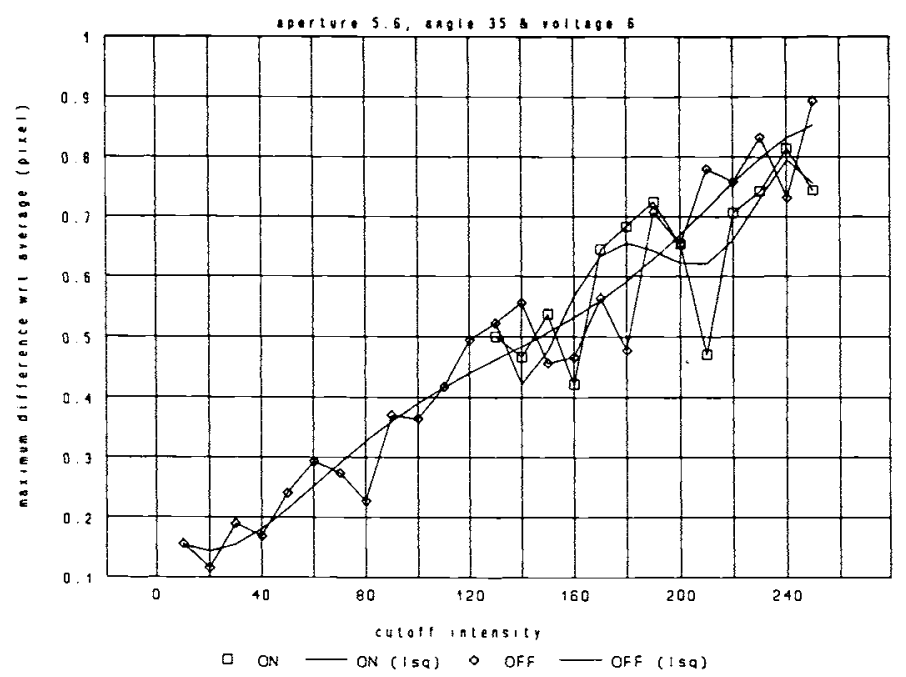

Fig. 2 Centroid locating sensitivity as function of cutoff intensity.

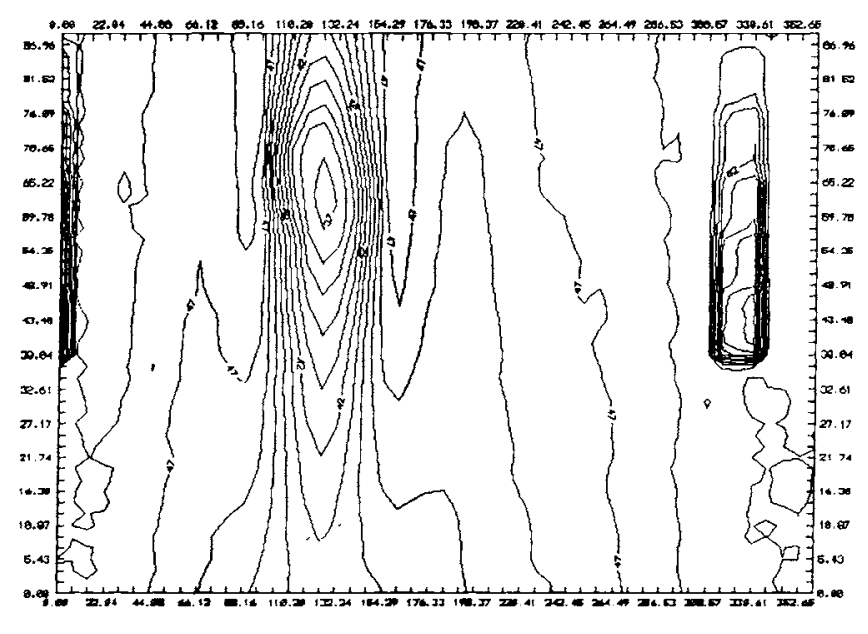

(a)

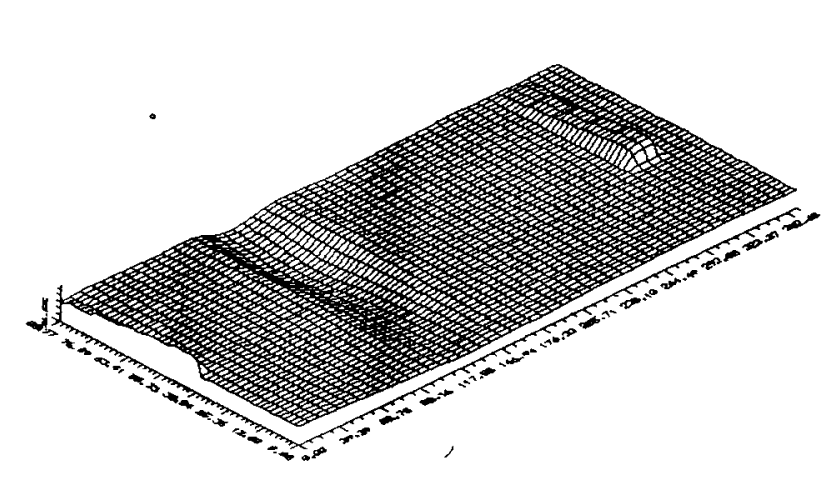

(b)
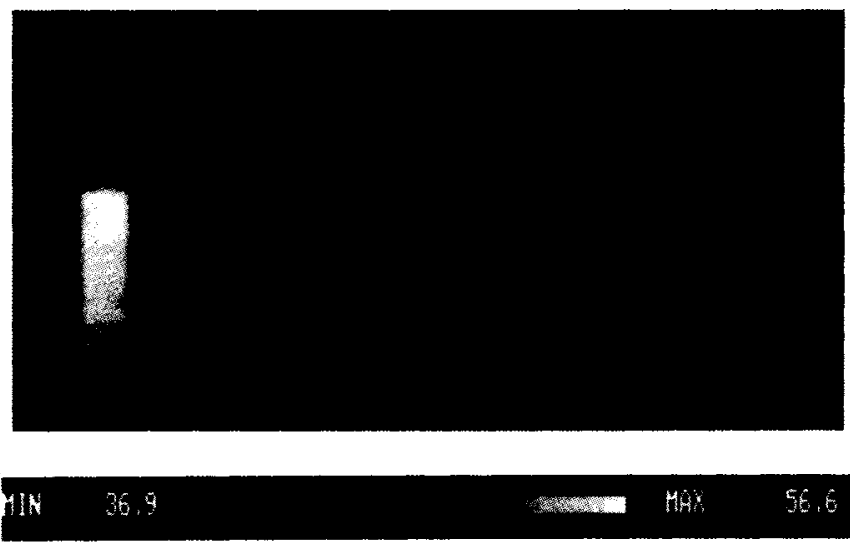

(c)

Fig. 3 (a) Contour map and (b) topographic profile. (c) Color-coded contours of unwrapped dented can. 


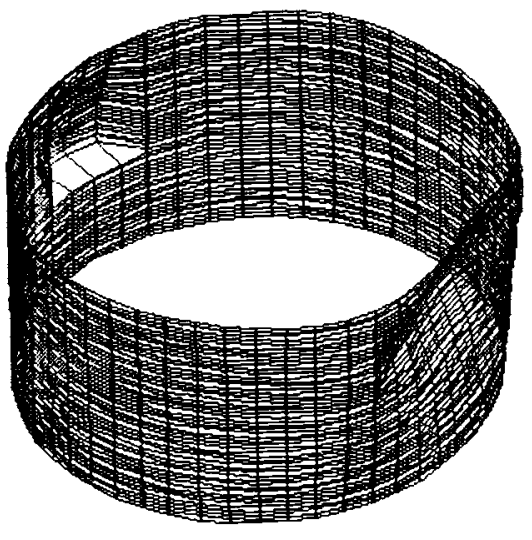

(a)

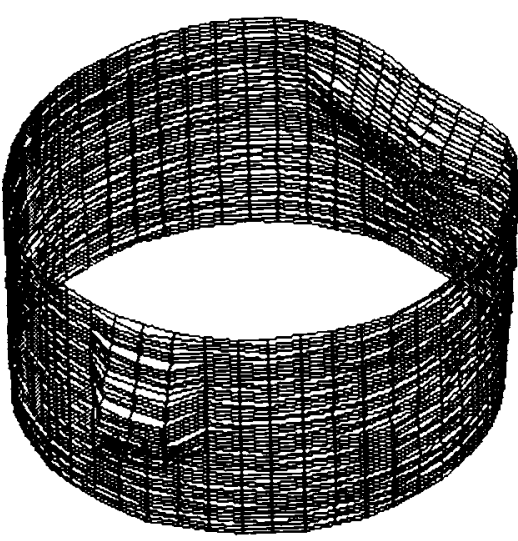

(b)

Fig. 4 Three-dimensional wire diagram views: (a) latitude 56 deg and longitude $-94 \mathrm{deg}$, and (b) latitude $-56 \mathrm{deg}$, and longitude $-80 \mathrm{deg}$.

slice while deviations inward indicate a dent and deviations outward show a protrusion. This is clearly noticeable in the figure.

The ubiquitous mannequin head was the second object evaluated. As in the previous case, no special preparation of the surface was made prior to testing. Figure 6 depicts the unwrapped contour map as contour lines and also as a colorcoded map. A much better form of display in this case is the 3-D view, and the ability to animate it within the desktop computer provides for added versatility. Figure 7 shows three discrete views of the mannequin head. Finally, Fig. 8 displays the data as slices taken at various heights from the top of the mannequin head. There is obviously no rotational symmetry in this case as was present for the can. The profile identifying the nose and the one with the nose and ears are unmistakable.

\section{Projected Grating Method}

Although the previous scheme can be thought of as a projected grid method wherein each bright line is analyzed as it is generated, this section describes an exciting and cheaper alternative to generating a projected grid on the surface of the can as it is "unwrapped." This scheme allows for qualitative assessment to be made rapidly for defect or damage assessment prior to any quantitative determination if desired. Indeed, using a digital imaging system, a moiré can be gen-
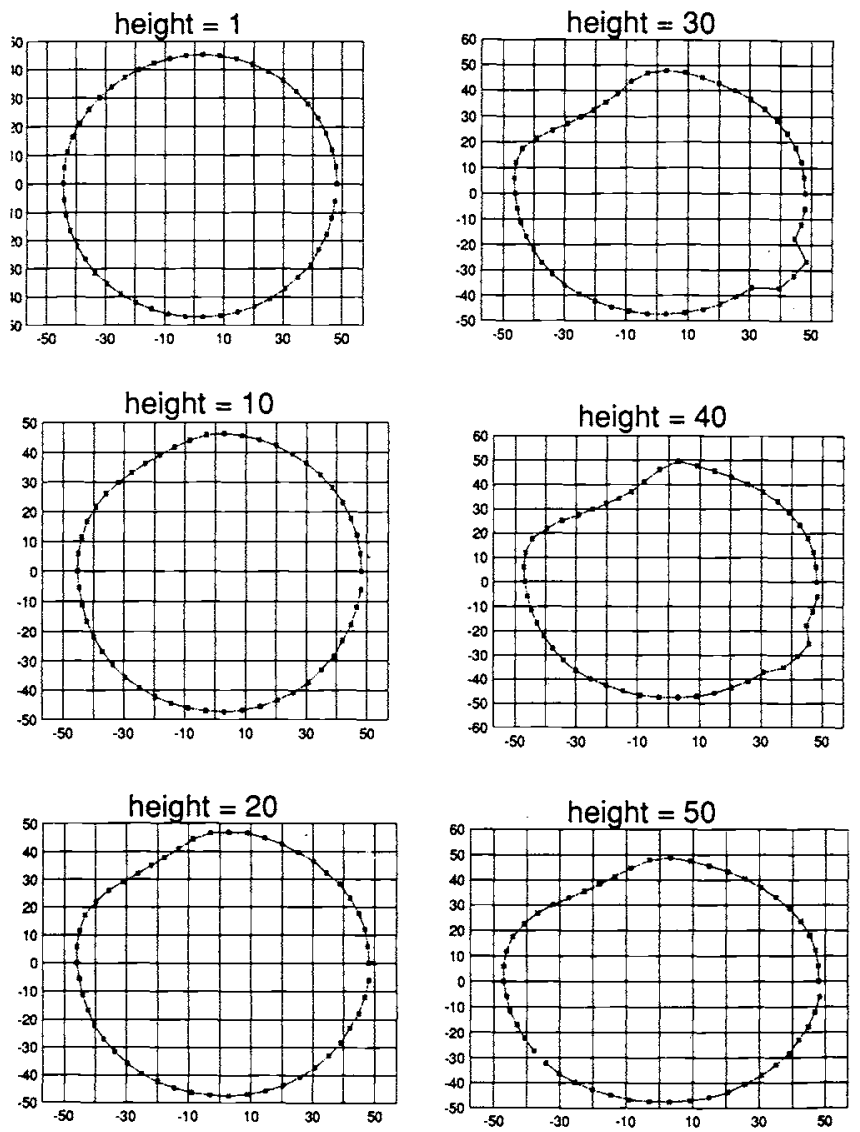

Fig. 5 Can slices at various heights from top to bottom.

erated while the projected grid is being scanned enabling immediate highlighting of defective areas.

The overall experimental schematic for this case is similar to the striped light system as seen in Fig. 9(a). There are, however, some differences in the individual components of the setup. The object, as before, is mounted on a rotational stage to enable 360-deg data acquisition. However, a simple variable speed turntable is sufficient for this purpose. There is no need of a step control stage. The laser diode with the stripe generating optics is identical to the previous setup. However, this stripe of light is further structured by placing a line grating to slice the stripe into dark and bright regions. In addition, the laser could operate in the pulsed mode with adjustable pulse width and pulse separation. As is demonstrated later, using the sliced strip light illumination will generate a horizontal grating on the unwrapped cylinder, while the pulse mode operation gives a vertical grating. Of course, both these modes could operate simultaneously resulting in a crossed projected grating. Furthermore, the laser diode is placed at an angle to both the vertical and horizontal planes passing through the viewing lens so that both gratings would be modulated by the depth profile of the object.

Finally, the imaging CCD camera operates in the so-called time delay and integration (TDI) mode. In this mode the camera functions much like the conventional analog drum 


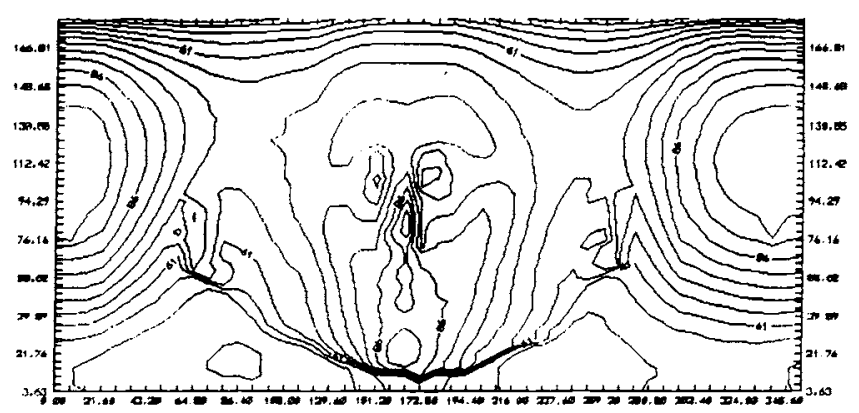

(a)

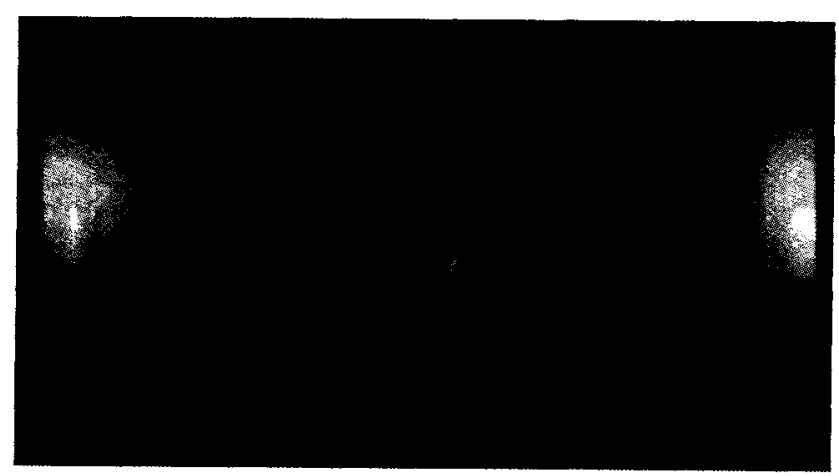

(b)

Fig. 6 (a) Contour map in the form of contour lines and (b) colorcoded map of a mannequin head.

(or periphery) camera. In a standard drum camera [Fig. 9(b)], each stripe of light is imaged onto the film that is moving laterally as the object rotates. One thus has the unwrapped image of the object. Using a striped light illumination precludes the need for a slit in front of the imaging lens. The speeds of the rotating object and the film translation must be matched to avoid stretching or compressing of the unwrapped object. The TDI mode of operation is the digital counterpart of the drum camera. As with digital imaging, there are no physically moving parts. In their stead the charge collection sites move from one end of the detector to the other [Fig. 9(c)] thus effectively simulating a moving film. The present CCD array has 192 pixels per line and 165 lines. In the TDI mode, the charge of each pixel on a line is added to itself as it moves from one end of the detector to the other. There is thus a time delay from the moment a line of pixels is first illuminated to the instant when it is read into the buffer and the charge of the pixel is integrated over the 165 lines. Thus the name TDI. The scanning speed of the camera, i.e., the speed at which the lines move from one end to the other, can be adjusted with the maximum being about 250,000 lines/ min or $0.25 \mathrm{~ms} /$ line and a maximum of 3000 lines can be buffered. Of course, digital storing enables one to save every $n$ 'th line, thus producing a scanning moiré effect.

\subsection{Experimental Results}

The two objects used in the first part were also evaluated using this system. The TDI camera was scanning at 50,000 lines $/ \mathrm{min}$, i.e., each line was imaged in $1.2 \mathrm{~ms}$. As mentioned earlier, depending on how the light stripe is further modulated

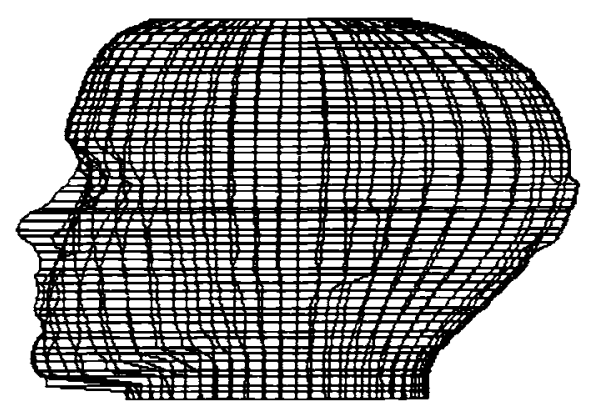

(a)

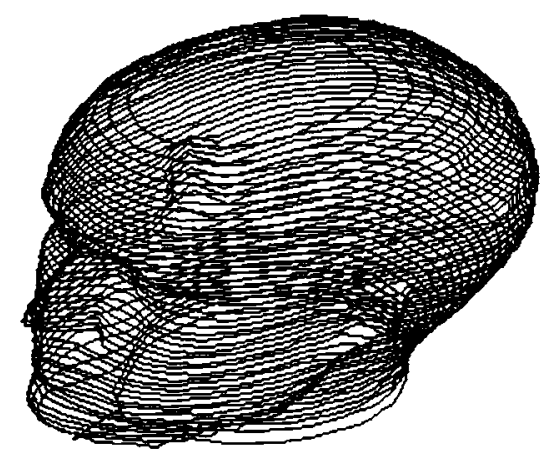

(b)

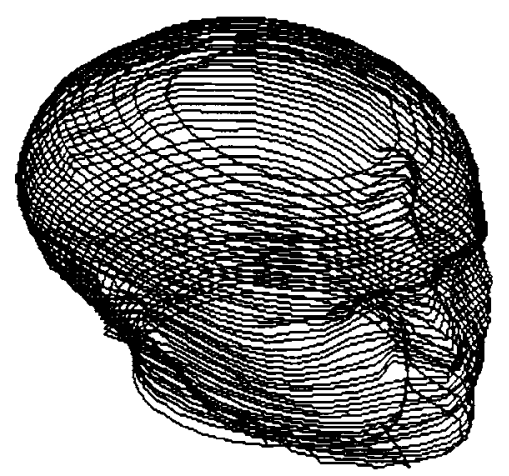

(c)

Fig. 7 Three-dimensional views of the profiled mannequin head: (a) latitude 0 deg and longitude 0 deg, (b) latitude $-40 \mathrm{deg}$ and longitude $45 \mathrm{deg}$, and (c) latitude $46 \mathrm{deg}$ and longitude $-116 \mathrm{deg}$.

(or structured), a horizontal, or vertical, or a crossed line grating can be generated. By slicing the light stripe using a grating, a horizontal projected grating is recorded on the unwrapped periphery of the can [Fig. 10(a)]. If the laser diode is used in the pulse mode a vertical grating will be projected as shown in Fig. 10(b), and using both these modulation techniques simultaneously gives a crossed grating as shown in Fig. 10(c). For the horizontal grating the pitch of the projected lines depends on the spacing of the lines of the grating used to slice the stripe. For the vertical grating, the pitch is a function of the stripe width and the pulse separation. The pulse width has no influence on the pitch, but to prevent smearing it is necessary to keep the pulse width to a value much smaller than the time required to image a line.

Figure 10 clearly shows deviations in the projected grating line due to the dent in the can. These distortions can be 

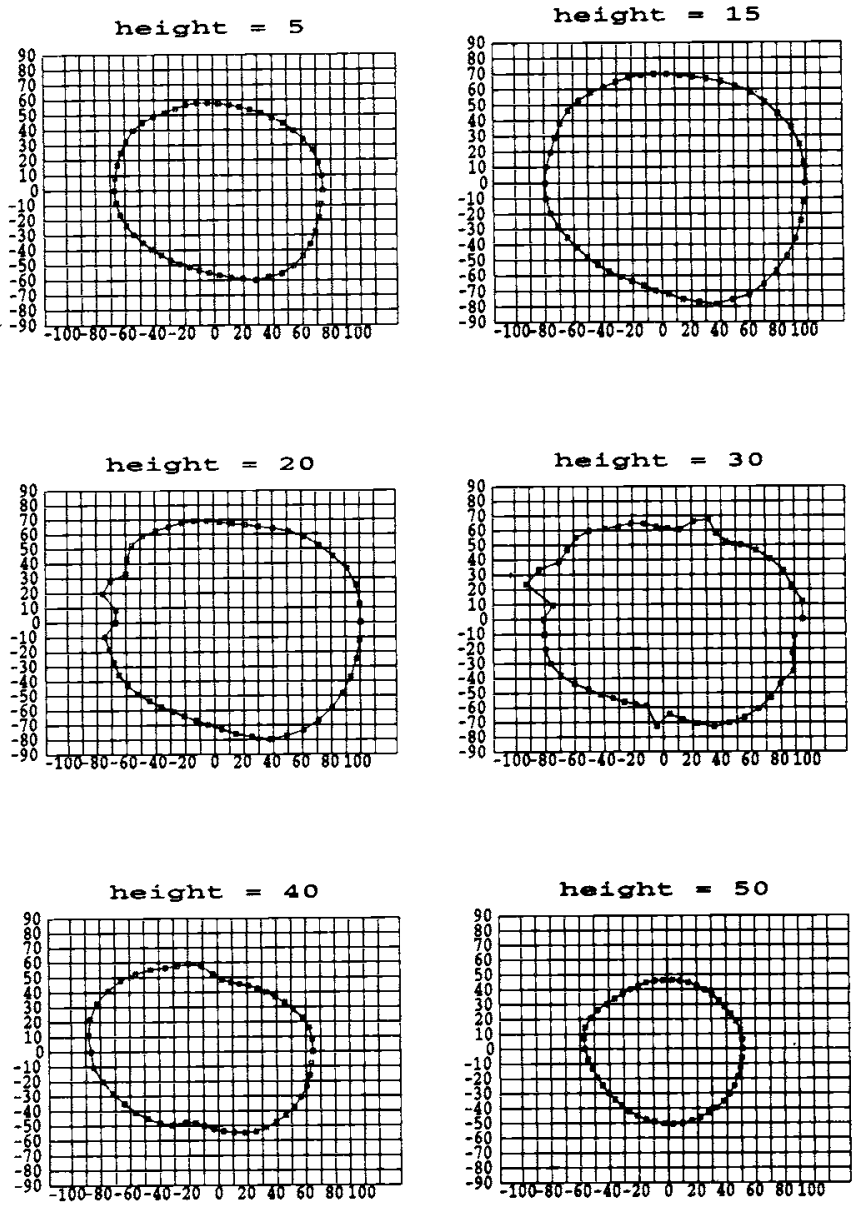

Fig. 8 Slices of the mannequin head profile at various heights from the top.

highlighted by subtracting the uniform pattern using the moiré effect. The moiré can be generated on-line by recording every $n$ 'th line, where $n$ corresponds to the average pitch of the recorded grating. Figure 11 (a) depicts such a moiré pattern where the distortions in the grating are now bold variations. Indeed, qualitatively the pattern is similar to Fig. 3(b). Only the vertical projected grating lines can be displayed in this manner and although the contours can be quantified, off-line grating processing is preferred. Various schemes exist for analyzing these projected grid patterns. In this article the phase shifting and logical moire (PSALM) ${ }^{15}$ routine is adopted. Briefly, PSALM invokes the following steps.

1. The projected grating is thresholded using a moving average scheme to produce a binary grating.

2. A computer-generated reference grating whose pitch, as a first choice, is the average spacing of the projected grating lines is formed. The user has a choice to select a different pitch; however, as long as this is not significantly different from the average spacing, this choice is immaterial.

3. A logical moire pattern is generated by using the logical AND, OR, or XOR operations on the above two gratings. While the AND and OR moiré patterns are similar to those obtained using physical superposition of real

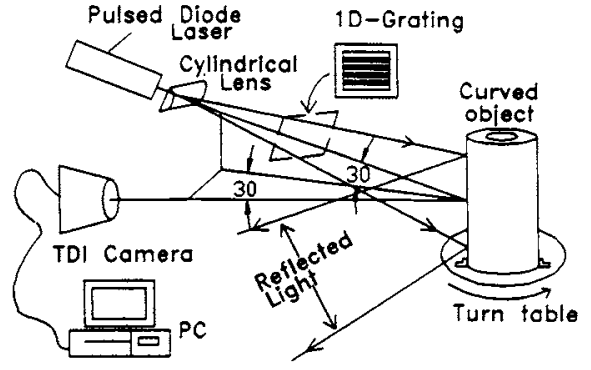

(a)

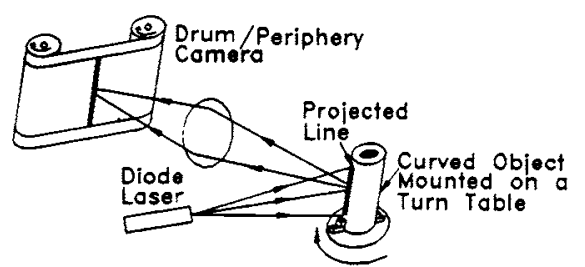

(b)

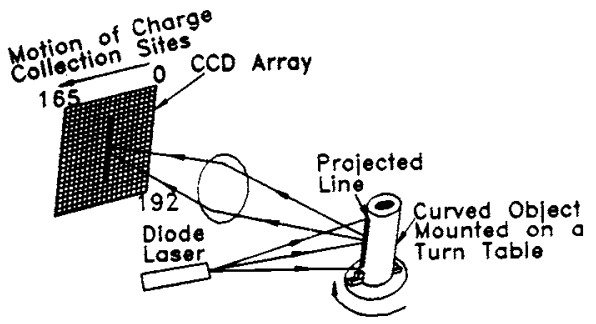

(c)

Fig. 9 (a) Schematic for the modulated structured light system, (b) imaging a moving object using the drum camera, and (c) time delay and integration mode of imaging.

gratings, the XOR gives an enhanced pattern. Figure 11 (b) is such a pattern.

4. The computer-generated reference grating is translated via software to obtain at least three, usually four, phaseshifted images. Before application of the appropriate phase shifting expression, ${ }^{16}$ the intensity from each image is averaged over the pitch of the reference grating. Furthermore, despite the fact that the phase-shift expressions are for sinusoidal intensity profiles, the error involved in using the triangular profile obtained in this case is small. ${ }^{17}$

5. The phase maps are unwrapped to overcome the modulo $2 \pi$ limit of the arctangent.

Figure 12 shows the resulting topographic and contour maps. PSALM is susceptible to noise as with any other phase unwrapping algorithm. In particular, thresholding enhances the noise. However, using thresholding with low-pass filtering ${ }^{18}$ noise can be significantly averted. Nevertheless, the contour maps do identify the dent quite well.

Figure 13 shows the gratings projected on the mannequin head and using the scanning moire, ${ }^{19,20}$ while recording produces the contour map shown in Fig. 14, which closely matches those of Fig. 6. 


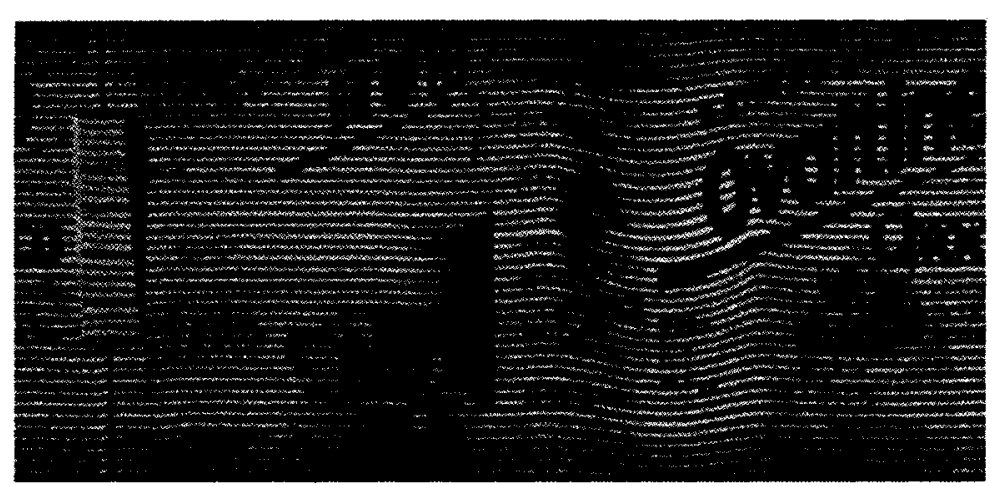

(a)

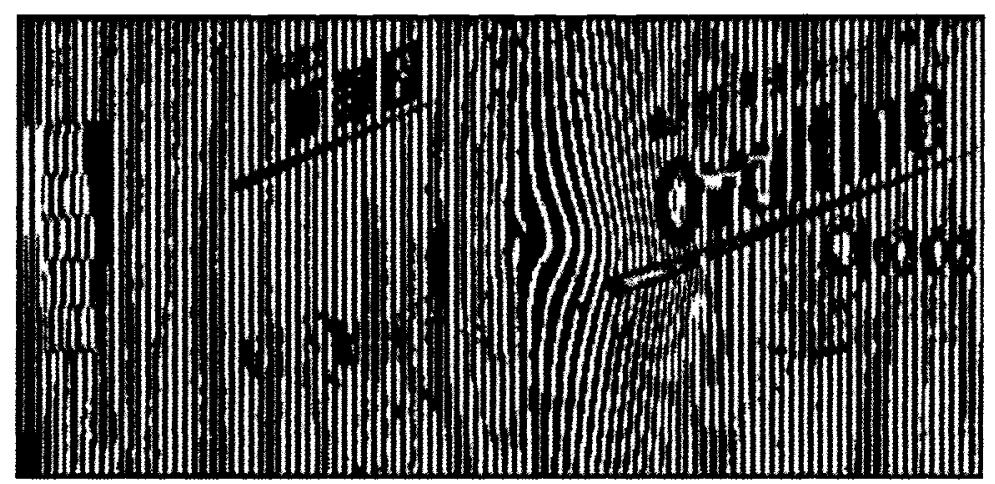

(b)

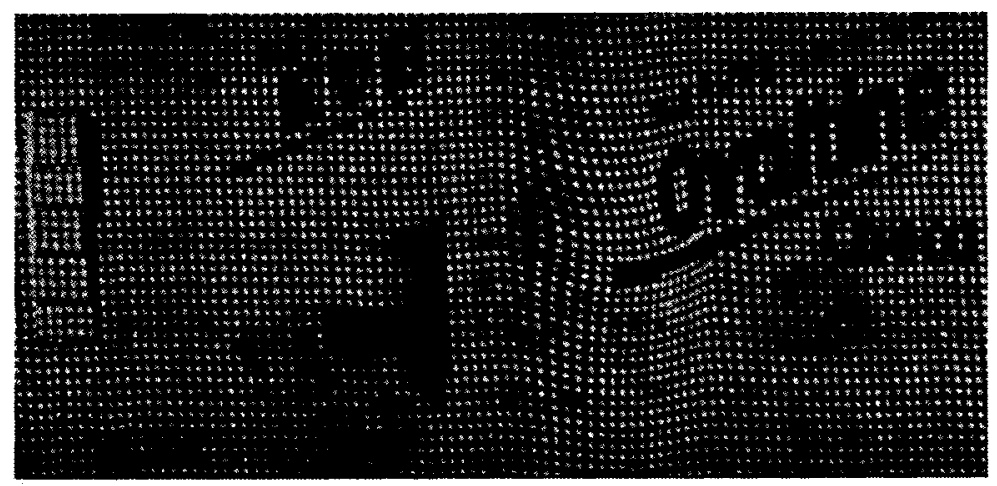

(c)

Fig. 10 (a) Horizontal, (b) vertical, and (c) crossed gratings using modulated structured light and TDI imaging.

\section{Conclusion}

Two optical methods for shape measurement and defect detection have been described and demonstrated. The first uses the familiar structured light system and evaluates each projected line before stepping to the next angle. The method has good sensitivity and is a reliable tool. Compromises have to be made to balance the speed of data acquisition, the sampling points, and the line detection algorithm. The second method uses modulated structured light illumination and a CCD camera operating in the TDI mode to first produce an unwrapped $360-$ deg view of the object with a projected grating overlaid. Modulation of the structured light stripe is accomplished by either placing a grating ahead of the line of light to slice it horizontally and/or by operating the laser diode in the pulse mode to generate the vertical grating or crossed grating lines. Analysis of these projected gratings are done off-line. However, for defect analysis a scanning moiré can be generated to highlight defects as bold variations. For quantitative analysis PSALM is proposed, although any one of the other methods can be equally applied. A future goal is to apply phase shifting directly to the distorted grating to further enhance the sensitivity and versatility of the second approach.

\section{Acknowledgments}

We wish to acknowledge the University Research Council and the Committee on Research and Conference for their support.

\section{References}

1. P. Cielo, "Optical techniques for industrial inspection," Academic Press (1988).

2. R. J. Ahlers, "Stereoscopic vision-an application oriented overview," Optics, Illumination, and Image Sensing for Machine Vision IV, Proc. SPIE, 1194, 298-308 (1989). 


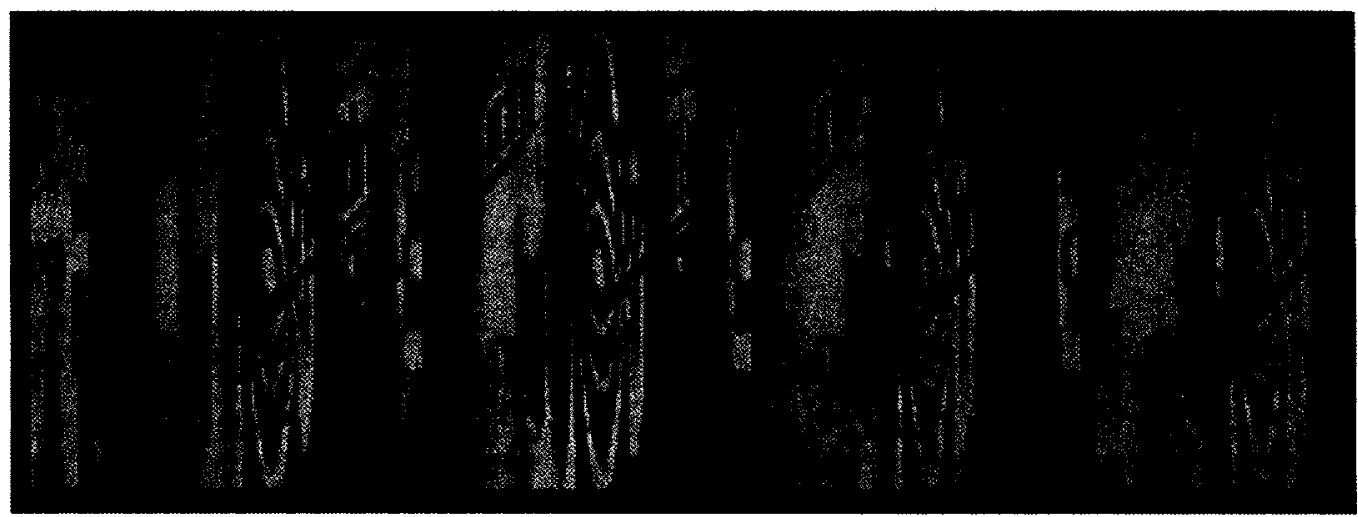

(a)

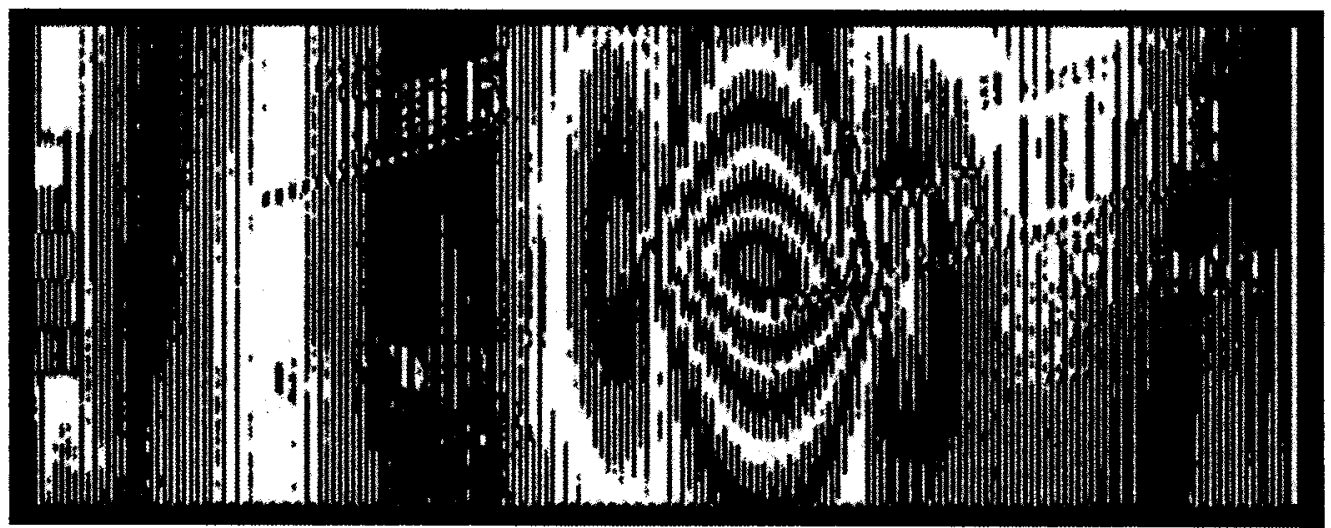

(b)

Fig. 11 (a) Scanning moiré by recording every fourth line from Fig. 10(b). (b) Logical XOR moiré of projected grating in Fig. 10(b) and computer-generated reference grating of pitch equal to four pixels.

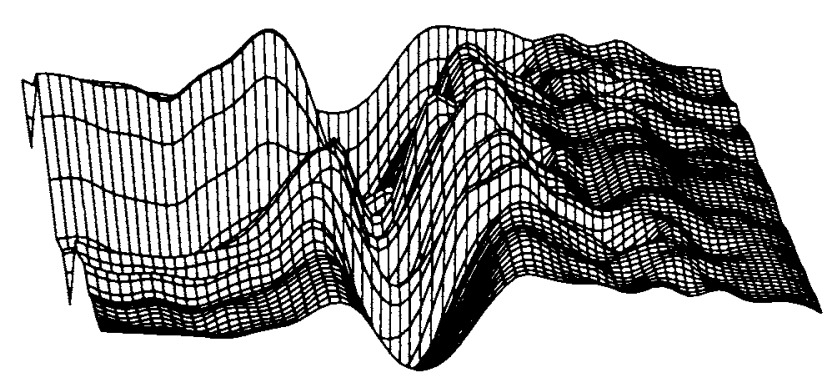

(a)

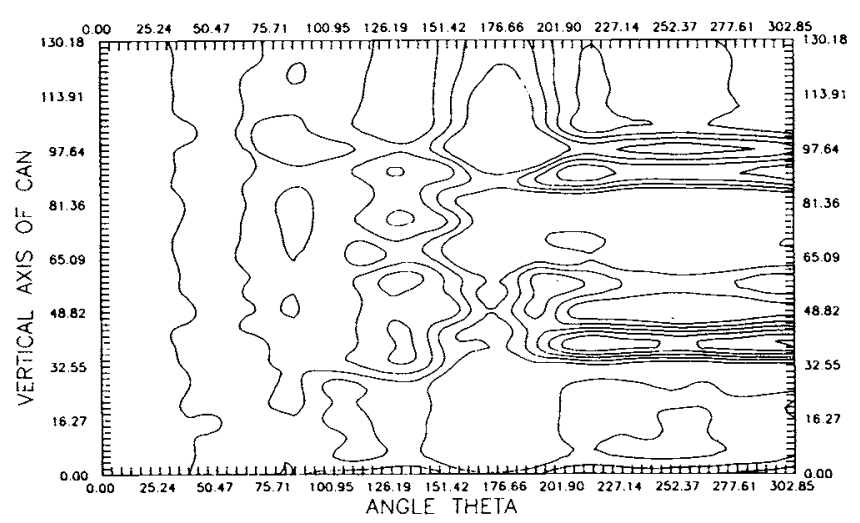

(b)

Fig. 12 (a) Topographic and (b) contour maps of dented can using PSALM.
3. V. Srinivasan, H. C. Liu, and M. Halioua "Automated phase measuring profilometry of 3-D diffuse objects,"' Appl. Opt. 23, 3105-3108 (1984). 4. M. Takeda and K. Mutoh, "Fourier transform profilometry for the automatic measurement of 3-D object shapes," Appl. Opt. 22, 3977-3982 (1983)

5. K. Sato and S. Inokuchi, "Range imaging system utilizing nematic liquid crystal mask," Proc. 1st Int. Conf. on Computer Vision, IEEE, pp. 657-661 (1987).

6. B. G. Batchelor, Ed., Automated Visual Inspection, IFS Publications Ltd., North Holland, U.K. (1985).

7. C. Torras. Ed., Computer Vision-Theory and Industrial Application, Springer Verlag, Berlin, New York (1992).

8. M. Halioua, R. S. Krishnamurthy, H. Liu, and F. P. Chiang, "Projection moire with moving gratings for automated 3D topography," Appl. Opt. 22(6), 850-855 (1983).

9. G. T. Reid, R. C. Rixon, and H. J. Messer, "Absolute and comparative measurements of three dimensional shape by phase measuring topography," Optics and Laser Technology 16, 315-319 (1984).

10. A. Asundi, "Moiré methods using computer generated gratings," Opt. Eng. 32(1), 107-116 (1993).

11. M. Halioua, R. S. Krishnamurthy, H. Liu, and F. P. Chiang, "Automated $360^{\circ}$ profilometry of 3-D diffuse objects," Appl. Opt. 24(14) 2193-2196 (1985).

12. X. X. Cheng, X. Y. Su, and L. R. Guo, "Automated measurement method for $360^{\circ}$ profilometry of diffuse objects,"' Appl. Opt. 30(10), 1274-1278 (1991).

13. C. G. Saunders, "Replication from 360 degree moire sensing," in Moire Fringe Topography and Spinal Deformity, M. S. Moreland, M. H. Pope, and G. W. D. Armstrong, Eds., Pergamon Press, New York (1981).

14. A. Asundi and Z. Haibo, "2D phase measurement using phase shifting and logical moire," Proc. SEM 50th Ann. Conf. on Exp. Mechanics, Dearborn, MI (1993)

15. A. Asundi and K. H. Yung," "Phase shifting and logical moire," J. Opt. Soc. Am. 8(10), 1591-1600 (1991).

16. K. Creath, "Phase measurement interferometry techniques," Progress in Optics, Vol. 26, E. Wolf, Ed., pp. 350-393, North Holland, Amsterdam (1989). 


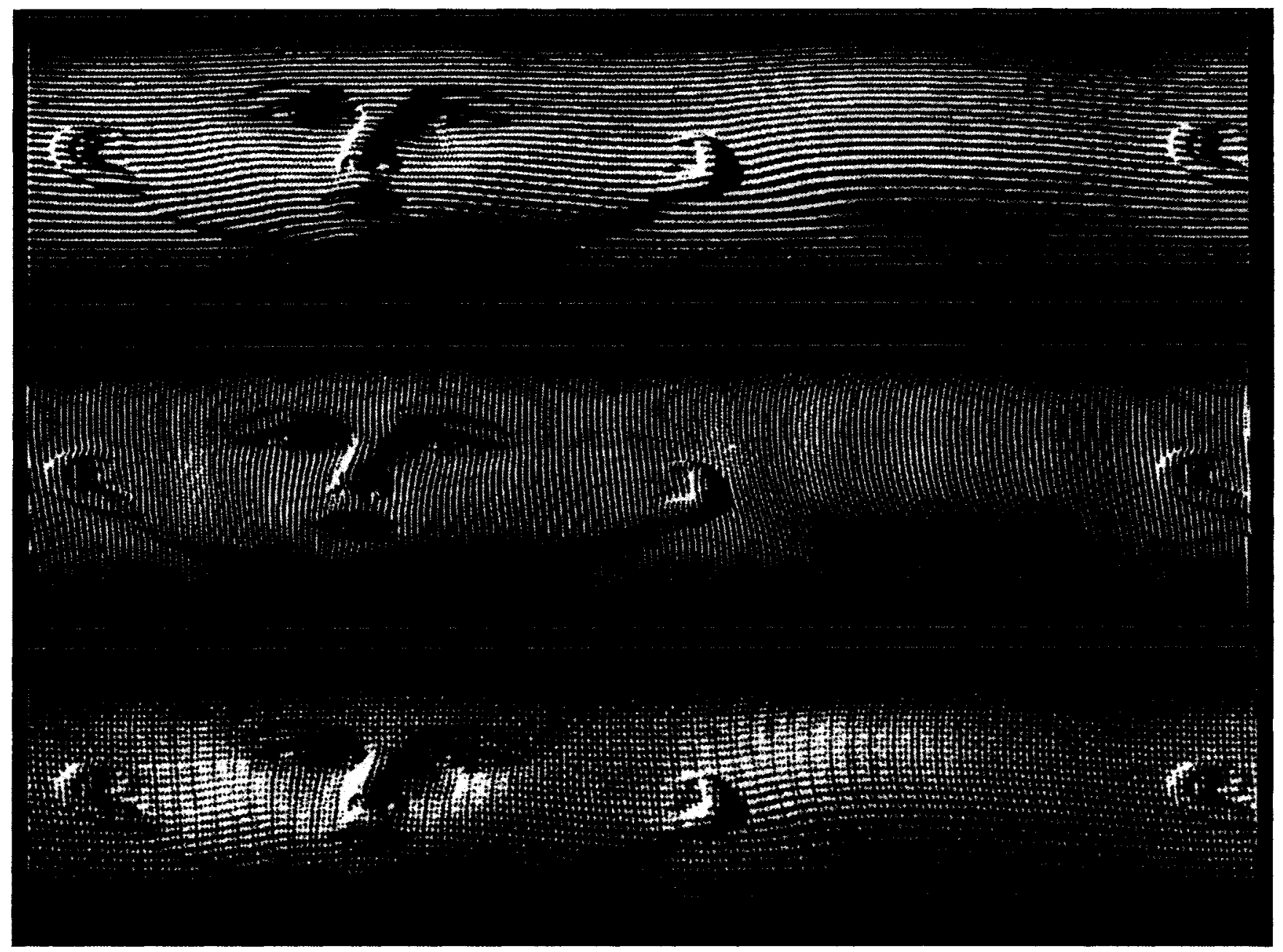

Fig. 13 Projected grating lines on unwrapped mannequin head.

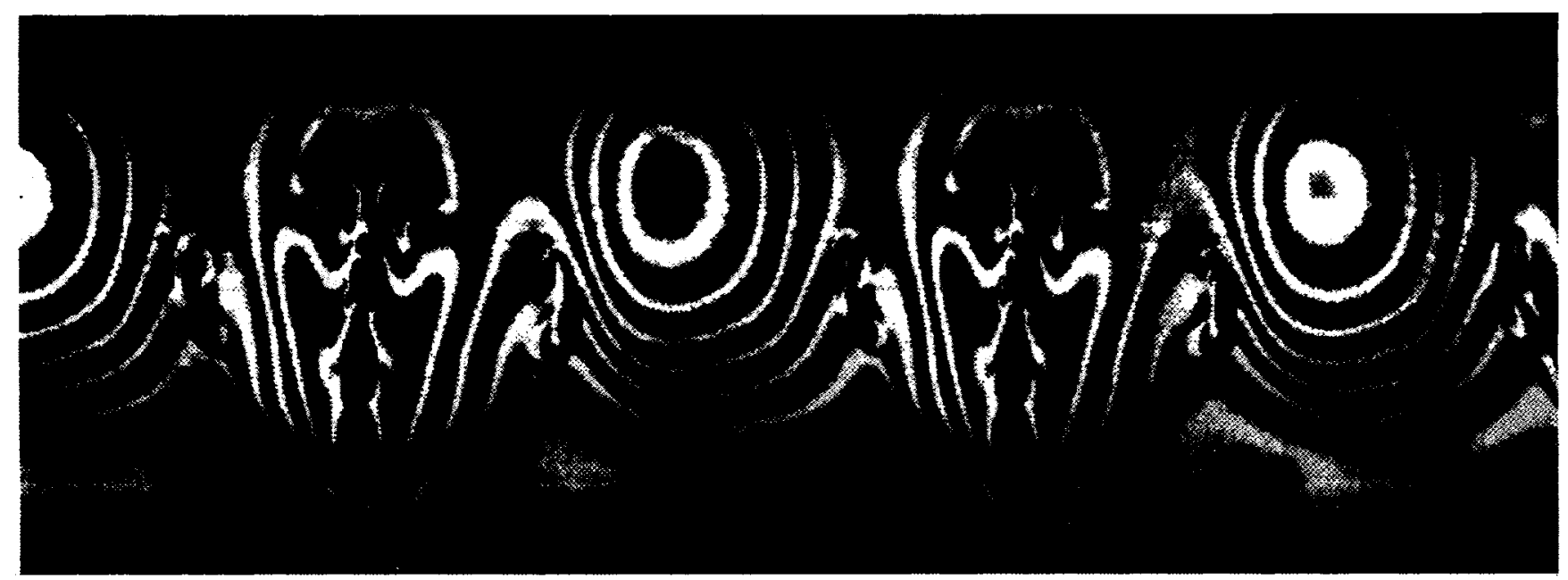

Fig. 14 Scanning moire of the projected grating to depict contours of depth. 
17. C. S. Chan and A. Asundi, "Structured-light-assisted CAD/CAM," Proc. SPIE 1713, 128-138 (1992).

18. A. Asundi, "Projection moire using PSALM," Photomechanics and Speckle Metrology II, F. P. Chiang, Ed., Proc. SPIE 1554, 257-265 (1991).

19. M. Idesawa, T. Yatagai, and T. Soma, "Scanning moire method and automatic measurement of 3-D shapes," Appl. Opt. 16, 2152-2162 (1977)

20. B. W. Bell and C. L. Koliopoulos, "Moire topography, sampling theory, and charged-coupled devices," Opt. Lett. 9, 171-173 (1984).

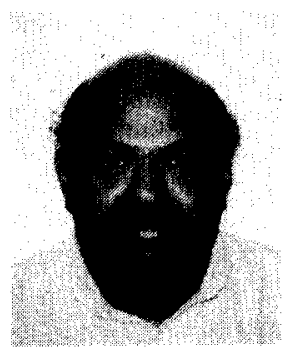

Anand K. Asundi graduated from IIT Bombay and subsequently received his PhD from the State University of New York at Stony Brook. Following a brief tenure as a research associate at Virginia Tech., he joined the University of Hong Kong where he is currently a senior lecturer. His research interests are in experimental mechanics, image processing, and machine vision, composite materials, and fracture and damage mechanics. He has published and presented more than 80 papers in these areas. He is a member of SPIE, OSA, SEM, ASME, and IMechE.

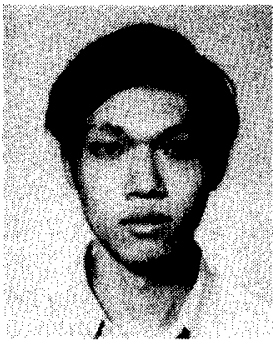

C. S. Chan received his BS degree in mechanical engineering from the University of Hong Kong in 1991. He is currently pursuing his MPhil degree at the same university. His research interests include $3-D$ shape measurement, machine vision, and related applications.

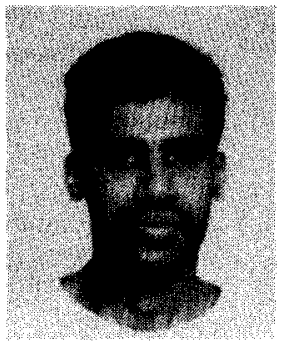

M. R. Sajan received his MS in optical instrumentation from the Indian Institute of Science. Currently he is pursuing a $\mathrm{PhD}$ in the Mechanical Engineering Department, University of Hong Kong. $\mathrm{He}$ is interested in optical interferometry, dynamic visualization, photomechanics, and automated inspection. 